\title{
Neutron Stars Exclude Light Dark Baryons
}

\author{
David McKeen, ${ }^{1,2, *}$ Ann E. Nelson, ${ }^{3, \dagger}$ Sanjay Reddy, ${ }^{4, \$}$ and Dake Zhou ${ }^{3,4, \S}$ \\ ${ }^{1}$ Pittsburgh Particle Physics, Astrophysics, and Cosmology Center, Department of Physics and Astronomy, University of Pittsburgh, \\ Pittsburgh, Pennsylvania 15260, USA \\ ${ }^{2}$ TRIUMF, 4004 Wesbrook Mall, Vancouver, British Columbia V6T 2A3, Canada \\ ${ }^{3}$ Department of Physics, University of Washington, Seattle, Washington 98195, USA \\ ${ }^{4}$ Institute for Nuclear Theory, University of Washington, Seattle, Washington 98195, USA
}

(Received 26 February 2018; revised manuscript received 1 June 2018; published 6 August 2018)

\begin{abstract}
Exotic particles carrying baryon number and with a mass of the order of the nucleon mass have been proposed for various reasons including baryogenesis, dark matter, mirror worlds, and the neutron lifetime puzzle. We show that the existence of neutron stars with a mass greater than $0.7 M_{\odot}$ places severe constraints on such particles, requiring them to be heavier than $1.2 \mathrm{GeV}$ or to have strongly repulsive self-interactions.
\end{abstract}

DOI: 10.1103/PhysRevLett.121.061802

Introduction.-Exotic states that carry baryon number and have masses below a few $\mathrm{GeV}$ have been theorized in a number of contexts, such as asymmetric dark matter [1,2], mirror worlds [3], neutron-antineutron oscillations [4], or nucleon decays [5]. In general, such states are highly constrained, because they can drastically alter the properties of normal baryonic matter-in particular, if too light, they can potentially render normal matter unstable. We currently understand that matter is observationally stable, because the standard model (accidentally) conserves baryon number. This ensures that the proton, the lightest baryon, does not decay (up to effects caused by higherdimensional operators that violate baryon number).

Now, consider the simple case of an electrically neutral single new fermion $\chi$ that carries a unit baryon number and carries no other conserved charge. Assuming that its couplings to ordinary matter are not highly suppressed, because of the conservation of baryon number and electric charge, it must have a mass larger than the difference between the proton and electron masses, $m_{\chi}>m_{p}-$ $m_{e}=937.76 \mathrm{MeV}$, in order to not destabilize the proton. In fact, a slightly stronger lower bound on $m_{\chi}$ comes from the stability of the weakly bound ${ }^{9} \mathrm{Be}$ nucleus: $m_{\chi}>$ $937.90 \mathrm{MeV}$. If $m_{\chi}>m_{n}=939.57 \mathrm{MeV}$, a new neutron decay channel can open up, $n \rightarrow \chi+\cdots$, where the ellipsis includes other particles that allow the reaction to conserve (linear and angular) momentum.

Published by the American Physical Society under the terms of the Creative Commons Attribution 4.0 International license. Further distribution of this work must maintain attribution to the author(s) and the published article's title, journal citation, and DOI. Funded by SCOAP ${ }^{3}$.
It is interesting to note that, if $m_{\chi}<m_{p}+m_{e}=$ $938.78 \mathrm{MeV}, \chi$ is itself kept stable by the conservation of baryon number and electric charge. It could therefore be a potential candidate for dark matter, which we know to be electrically neutral and stable. It is compelling that in such a situation the stability of normal matter and of dark matter is ensured by the same symmetry: baryon number.

A potential new decay channel for the neutron has recently received attention as a solution to the $4 \sigma$ discrepancy between values of the neutron lifetime measured using two different techniques, the "bottle" and "beam" methods $[3,6,7]$. The bottle method, which counts the number of neutrons that remain in a trap as a function of the time and is therefore sensitive to the total neutron width, gives $\tau_{n}^{\text {bottle }}=879.6 \pm 0.6 \mathrm{~s}$ [8]. The beam method counts the rate of protons emitted in a fixed volume by a beam of neutrons, thus measuring only the $\beta$-decay rate of the neutron, and results in $\tau_{n}^{\text {beam }}=888.0 \pm 2.0 \mathrm{~s}$ [9]. These two measurements can be reconciled by postulating a new decay mode for the neutron, such as $n \rightarrow \chi+\cdots$, with a branching fraction

$$
\mathrm{Br}_{n \rightarrow \chi}=1-\frac{\tau_{n}^{\text {bottle }}}{\tau_{n}^{\text {beam }}}=(0.9 \pm 0.2) \times 10^{-2}
$$

However, a recent reevaluation of the prediction for the neutron lifetime from post-2002 measurements of the neutron $g_{A}$ concludes that any nonstandard branching for the neutron is limited to less than $2.7 \times 10^{-3}$ at $95 \%$ C.L. [10].

In this Letter, we note that a new state that carries baryon number and has a mass close to the neutron's can drastically affect the properties of nuclear matter at densities seen in the interiors of neutron stars. In neutron stars, the neutron chemical potential can be significantly larger than $m_{n}$, reaching values of $\simeq 2 \mathrm{GeV}$ in the heaviest neutron 
stars [11]. Thus, any exotic particle that carries baryon number and has a mass $\lesssim 2 \mathrm{GeV}$ will have a large abundance if in chemical equilibrium. Because they replace neutrons, their presence will soften the equation of state of dense matter by reducing the neutron Fermi energy and pressure while contributing to an increase in the energy density. This will, in turn, reduce the maximum mass of neutron stars from those obtained using standard equations of state for nuclear matter. As we shall show below, even a modest reduction in the pressure at a high density can dramatically lower the maximum mass to a value that is significantly smaller than the observed heaviest neutron stars with masses $\simeq 2 M_{\odot}[12,13]$.

The remainder of this Letter is organized as follows. In Sec. II, we describe a simple model of fermion dark matter which is charged under baryon number. In Sec. III, we show the results of a computation of the effects of such a fermion on the mass-radius relation and maximum mass of neutron stars. Possible extensions of these constraints, future work, and ways to avoid the constraints are described in the conclusions, Sec. IV.

Model. - We begin by considering a simple model with a single neutral Dirac fermion $\chi$ that carries a unit baryon number. As mentioned above, $m_{\chi}>937.90 \mathrm{MeV}$ so as to not destabilize ${ }^{9} \mathrm{Be}$. The relevant terms in the effective Lagrangian involving the neutron are

$$
\mathcal{L}_{\text {eff }}=\bar{n}\left(i \not \partial-m_{n}\right) n+\bar{\chi}\left(i \not \partial-m_{\chi}\right) \chi-\delta(\bar{\chi} n+\bar{n} \chi),
$$

where $\delta$ is a coupling determined by the underlying theory. A simple UV completion $[4,14]$ of this involves integrating out a scalar diquark coupled to $u$ and $d$ quarks as well as to $d$ and $\chi$, generating the four-fermion operator

$$
\frac{1}{\Lambda^{2}} \bar{\chi} u d d
$$

Matching this onto the effective theory gives

$$
\delta \sim \frac{0.01 \mathrm{GeV}^{3}}{\Lambda^{2}} .
$$

In what follows, we assume that this coupling between $n$ and $\chi$ is small, in particular, $|\delta| \ll|\Delta m|$, where $\Delta m \equiv m_{n}-m_{\chi}$. This coupling leads to a mixing between $n$ and $\chi$, and the mass terms are diagonalized by taking $n \rightarrow n+\theta \chi$, $\chi \rightarrow \chi-\theta n$, where the mixing angle is $\theta=\delta / \Delta m$.

If $m_{\chi}<m_{n}$, a new decay mode for the neutron opens up, $n \rightarrow \chi \gamma$. In addition, if $m_{\chi}<m_{p}+m_{e}=938.78 \mathrm{MeV}, \chi$ is stable. The new decay mode for the neutron comes from the neutron magnetic dipole moment operator, which, after the mass matrix is diagonalized, contains the term

$$
\mu_{n} \theta \bar{\chi} \sigma^{\mu \nu} n F_{\mu \nu},
$$

where $\mu_{n}=-1.91 e /\left(2 m_{p}\right)=-0.31 \mathrm{GeV}^{-1}$ is the neutron magnetic dipole moment. The partial width for $n \rightarrow \chi \gamma$ is

$$
\Gamma_{n \rightarrow \chi \gamma}=\frac{\mu_{n}^{2} \theta^{2} m_{n}^{3}}{16 \pi}\left(1-\frac{m_{\chi}^{2}}{m_{n}^{2}}\right)^{3} \simeq \frac{\mu_{n}^{2} \theta^{2} \Delta m^{3}}{2 \pi} .
$$

Given a total width of $\Gamma_{n}=1 / \tau_{n}^{\text {bottle }}=(879.6 \mathrm{~s})^{-1}$, the branching ratio for the neutron to decay into $\chi \gamma$ is

$$
\begin{aligned}
\mathrm{Br}_{n \rightarrow \chi \gamma} & =0.01\left(\frac{\Delta m}{1 \mathrm{MeV}}\right)^{3}\left(\frac{\theta}{7 \times 10^{-10}}\right)^{2} \\
& =0.01\left(\frac{\Delta m}{1 \mathrm{MeV}}\right)\left(\frac{\delta}{7 \times 10^{-13} \mathrm{GeV}}\right)^{2} .
\end{aligned}
$$

Thus, we see that, for $m_{n}-m_{\chi} \sim 1 \mathrm{MeV}$, a mixing angle of the order of $10^{-9}$, or a $n-\chi$ coupling of about $10^{-12} \mathrm{GeV}$, can explain the neutron lifetime anomaly. (We note here why a model with Dirac $\chi$ where baryon number is conserved is necessary. If instead $\chi$ were Majorana with $\theta=10^{-9}$ and $\Delta m=1 \mathrm{MeV}$, a $\Delta B=2 n-\bar{n}$ transition amplitude of roughly $\theta^{2} \Delta m \sim 10^{-21} \mathrm{GeV}$ would arise. This is many orders of magnitude larger than the experimental upper bound of $10^{-33} \mathrm{GeV}$.) This value of $\delta$ corresponds to a scale for the four-fermion interaction in Eq. (3) of $\Lambda \sim 10^{5} \mathrm{GeV}$. We note here, however, that a very recent search for the decay $n \rightarrow \chi \gamma$ using ultracold neutrons sets a limit on this branching, for $937.90 \mathrm{MeV}<m_{\chi}<$ $938.78 \mathrm{MeV}$, of roughly $10^{-3}$ [15].

Although $\delta \sim 10^{12} \mathrm{GeV}$ is a small coupling between the neutron and $\chi$, it can lead to the efficient conversion of neutrons into $\chi$ 's in the high-density environments encountered inside neutron stars. In addition, because of the large neutron chemical potential inside neutron stars, the conversion $n \rightarrow \chi$ can take place there even for $m_{\chi}>m_{n}$, where free neutron decays are kinematically blocked.

We investigate the effects of a $\chi-n$ coupling on neutron stars in the next section.

Neutron stars.-The structure of neutron stars is determined by the equation of state (EOS) of dense matter which specifies the relationship between pressure $P$ and energy density $\epsilon$. For a given EOS, $P(\epsilon)$, the Tolman-OppenheimerVolkoff (TOV) equations of general relativistic hydrostatic structure can be solved numerically to obtain the mass-radius curves $[16,17]$. Although there remain large uncertainties associated with the EOS at a supranuclear density, the EOS up to nuclear saturation density $n_{s} \simeq 0.16 \mathrm{fm}^{-3}$ can be calculated using nuclear Hamiltonians and the nonrelativistic quantum many-body theory to obtain $P_{\text {nuc }}\left(\epsilon_{\text {nuc }}\right)$ [18-20]. Furthermore, absent phase transitions to new states of matter, modern nuclear EOSs are able to estimate uncertainties associated with the extrapolation to high density, since they account for two- and three-body nuclear forces consistently and are based on a systematic operator expansion rooted in the effective field theory [21]. Representative nuclear 


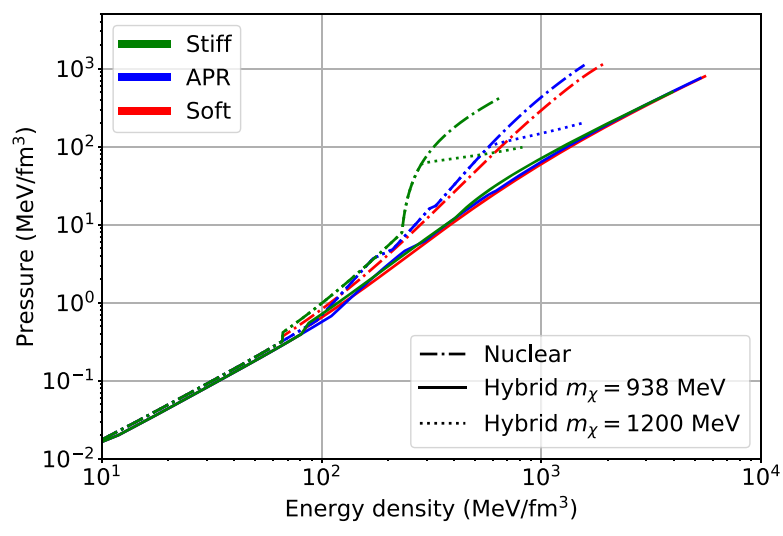

FIG. 1. Hybrid EOS and underlying nuclear EOS. The standard nuclear matter is shown as dash-dotted curves. The stiff EOS makes a second-order transition to a causal EOS at $n_{B}=1.5 n_{s}$. This is the stiffest possible EOS and predicts a maximum mass of $\simeq 3.3 M_{\odot}$ (Fig. 2). Adding a dark baryon with $m_{\chi}=938 \mathrm{MeV}$ results in solid curves, which are dominated by $\chi$ 's Fermi gas EOS for $\epsilon \gtrsim 0.1 \mathrm{MeV} / \mathrm{fm}^{3}$. Dotted lines show a hybrid EOS with $m_{\chi}=1.2 \mathrm{GeV}$. All curves are truncated at maximum central densities inside stable neutron stars.

EOSs are shown as dot-dashed curves in Fig. 1. The curve labeled APR, calculated by Akmal, Pandharipande, and Ravenhal [18], has been widely used to describe neutron stars. The curves labelled "soft" and "stiff" are the extreme possibilities consistent with our current understanding of uncertainties associated with the nuclear interactions up to $1.5 n_{s}[19,20]$. The EOS labeled soft uses a nuclear EOS with a low pressure compatible with neutron matter calculations and is extrapolated to a high density to ensure that produces a neutron star with a mass just shy of $2 M_{\odot}$. The curve labeled stiff is obtained by using the largest pressure up to $1.5 n_{s}$ compatible with neutron matter calculations, and at a higher density we use the maximally stiff EOS with $P(\epsilon)=P_{0}+$ $\left(\epsilon-\epsilon_{0}\right)$, where $P_{0}$ and $\epsilon_{0}$ are the pressure and energy density, respectively, predicted by the nuclear EOS at $1.5 n_{s}$. We believe that the soft and stiff EOSs bracket the extreme possibilities subject to constraints from nuclear physics and observations of the massive neutron stars with $M_{\mathrm{NS}} \simeq 2 M_{\odot}$. In what follows, we shall use these EOSs to demonstrate that, despite the uncertainty at a supranuclear density, the observation of neutron stars with mass $M_{\mathrm{NS}} \simeq 2 M_{\odot}$ rules out the existence of a weakly interacting dark matter candidate which carries baryon number and has a mass in the range $937.90 \mathrm{MeV}<m_{\chi}<938.78 \mathrm{MeV}$. In fact, we shall find that any such weakly interacting particle with mass $m_{\chi} \lesssim 1.2 \mathrm{GeV}$ can be robustly excluded.

In Fig. 2, we show the mass-radius curve for neutron stars predicted by the standard nuclear EOS as dash-dotted curves. The curves terminate at the maximum mass. For the maximally stiff EOS, the speed of sound in the high-density region $c_{s}=c$, and this construction produces the largest maximum mass of neutron stars compatible with nuclear physics.

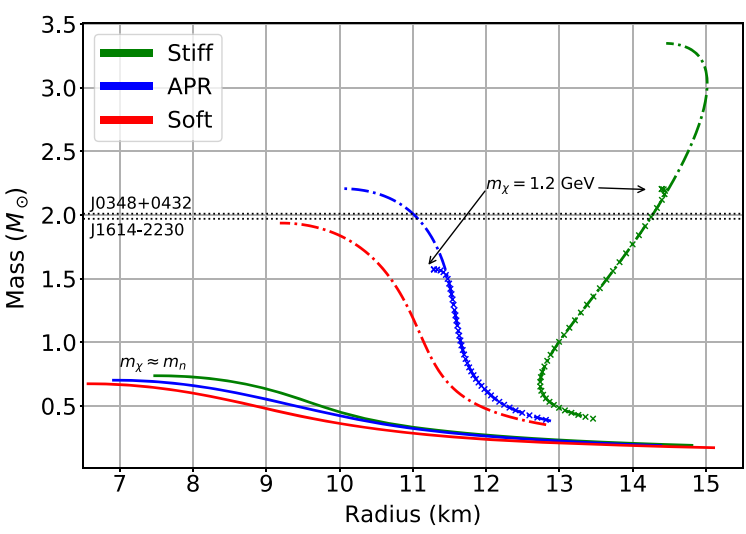

FIG. 2. The mass-radius relationship generated using the EOS in Fig. 1. Even for the extremely stiff EOS, the maximum mass of hybrid stars containing noninteracting dark neutrons does not exceed $0.8 M_{\odot}$. The measured masses of the two most massive neutron stars $\mathrm{J} 0348+0432$ and $\mathrm{J} 1614-2230$ are also shown.

Any exotic neutron decay channel $n \rightarrow \chi+\cdots$ which makes even a small contribution to the neutron width, of the order of the inverse lifetime of a neutron star, will be fast enough to ensure that $\chi$ is equilibrium inside the star. The typical age $t_{\mathrm{NS}}$ of old observed neutron stars is $t_{\mathrm{NS}} \approx 10^{6}-10^{8}$ years. In a dense medium, due to strong interactions, the dispersion relation of the neutron can be written as $\omega_{n}(p)=\sqrt{p^{2}+m_{n}^{2}}+\Sigma_{r}+i \Sigma_{i}$, where $\Sigma_{r}$ and $\Sigma_{i}$ are the real and imaginary parts, respectively, of its self-energy. The mixing angle is suppressed at a finite density and is given by

$$
\tilde{\theta}=\frac{\delta}{\sqrt{\Delta m^{2}+\Sigma_{i}^{2}}},
$$

where $\widetilde{\Delta m}=\Delta m+\Sigma_{r}$. Since $\Sigma_{r}$ and $\Sigma_{i}$ are expected to be of the order of $10-100 \mathrm{MeV}$ at the densities attained inside neutron stars [22], it is reasonable to expect the ratio $\tilde{\theta} / \theta$ to be in the range $0.01-0.1$. The rate of production of $\chi$ 's in the neutron star interior due to neutron decay, defined in Eq. (6), is suppressed by the factor $(\tilde{\theta} / \theta)^{2}$ but enhanced by $(\widetilde{\Delta m} / \Delta m)^{3}$ when $\widetilde{\Delta m}>\Delta m$. For $\widetilde{\Delta m} \approx 10 \mathrm{MeV}$, the neutron decay lifetime is $<10^{8} \mathrm{yr}$ when $\delta>10^{-19} \mathrm{GeV}$, and it is safe to assume that, for the phenomenologically interesting values of $\delta \simeq 10^{-14}-10^{-12} \mathrm{GeV}, \chi$ will come into equilibrium on a timescale $t \ll t_{\mathrm{NS}}$. (We delegate to future work a detailed calculation of the production rate for such small values of $\delta$ which may be interesting in other contexts).

Because $\chi$ carries baryon number, in equilibrium its chemical potential $\mu_{\chi}=\mu_{B}$, where $\mu_{B}$ is the baryon chemical potential. Given a nuclear EOS, the baryon chemical potential is obtained using the thermodynamic relation $\mu_{B}=\left(P_{\mathrm{nuc}}+\epsilon_{\mathrm{nuc}}\right) / n_{B}$, where $n_{B}$ is the baryon number density. If $\chi$ is a Dirac fermion with spin $1 / 2$ and its 
interactions are weak, its Fermi momentum and energy density are given by

$$
\begin{gathered}
k_{F \chi}=\sqrt{\mu_{B}^{2}-m_{\chi}^{2}}, \\
\epsilon_{\chi}=\frac{1}{\pi^{2}} \int_{0}^{k_{F \chi}} d k k^{2} \sqrt{k^{2}+m_{\chi}^{2}},
\end{gathered}
$$

respectively. The dark neutron number density $n_{\chi}=$ $k_{F \chi}^{3} / 3 \pi^{2}$ and its pressure $P_{\chi}=-\epsilon_{\chi}+\mu_{B} n_{\chi}$. The total pressure $P_{\text {tot }}=P_{\text {nuc }}+P_{\chi}$ and energy density $\epsilon_{\text {tot }}=\epsilon_{\text {nuc }}+$ $\epsilon_{\chi}$ are easily obtained, and the TOV equations are solved again to determine the mass-radius relation for hybrid stars containing an admixture of $\chi$ particles. The net result is a softer EOS where the pressure is lower at a given energy density, because, as we mentioned earlier, $\chi$ replaces neutrons and reduces their Fermi momentum and pressure. Results for $m_{\chi}=938 \mathrm{MeV}$ are shown in Fig. 2 as solid curves which terminate at the maximum mass. We allow the nuclear EOS to vary from maximally stiff to soft and also show the results for the APR EOS. The striking feature is the large reduction in the maximum mass. This reduction is quite insensitive to the nuclear EOS. Even for the maximally stiff EOS, the presence of noninteracting dark neutrons reduces the maximum mass to values well below observed neutron star masses. Thus, a dark neutron with $m_{\chi} \simeq 938 \mathrm{MeV}$ and weak interactions is robustly excluded. For larger $m_{\chi}$, we can still obtain useful bounds as long as $m_{\chi}$ is smaller than the baryon chemical potential attained in the core. For $m_{\chi}=1.2 \mathrm{GeV}$, we find, as expected, that the appearance of dark neutrons is delayed to a supranuclear density, but, as soon as they appear, they destabilize the star. This can be inferred by the behavior of the mass-radius relation labeled $m_{\chi}=1.2 \mathrm{GeV}$ and denoted by points represented as crosses. For the APR EOS the maximum mass is about $1.6 M_{\odot}$, and for the maximally stiff EOS it is about $2.2 M_{\odot}$.

Although interactions between $\chi$ 's and nucleons are necessarily weak, interactions between $\chi$ 's could be strong. [In the model leading to the four-fermion interaction of Eq. (3), $\chi$-nucleon interactions come from the four-fermion interaction $\bar{\chi} \chi \bar{d} d / \Lambda^{2}$. With $\Lambda \gtrsim 10^{5} \mathrm{GeV}$ for $\delta \lesssim 10^{-12} \mathrm{GeV}$, this interaction is highly suppressed compared to nuclear strength interactions.] If $\chi$ is charged under a $\mathrm{U}(1)$ with coupling strength $g$ to a new gauge boson with a mass $m_{V}$, repulsion between between $\chi$ 's modifies the EOS. In the mean field approximation, both the pressure and energy density are increased by

$$
\Delta P_{\chi}=\Delta \epsilon_{\chi}=\frac{1}{2} \frac{g^{2}}{m_{V}^{2}} n_{\chi}^{2} .
$$

For strong coupling with $g \simeq 1$ and small $m_{V}$ corresponding to a Compton wavelength of the gauge boson that is larger than the interparticle distance, this interaction energy will dominate. Under these conditions, the number density of $n_{\chi} \approx m_{V}^{2}\left(\mu_{B}-m_{\chi}\right) / g^{2}$ in equilibrium will be greatly reduced, and its impact on the dense matter EOS will be negligible. For $m_{\chi} \simeq m_{N}$, we find that when $g>$ $1.1\left(m_{V} / 100 \mathrm{MeV}\right)$ the stiff EOS predicts a maximum mass $>2 M_{\odot}$, while for the APR EOS we require $g>$ $2.4\left(m_{V} / 100 \mathrm{MeV}\right)$ to accommodate this maximum mass. Another possibility is that dark neutrons have interactions that mimic interactions between ordinary neutrons. In such a mirror scenario, we find that the maximum mass of neutron stars is $1.6 M_{\odot}$ for the APR EOS and $2.4 M_{\odot}$ for the maximally stiff EOS construction.

Conclusions. - States that carry baryon number and have a mass close to the nucleons have been studied in several scenarios. The extreme environments encountered in the interiors of neutron stars can readily produce such states. However, because these new states do not, in general, have the same interactions that neutrons do, they can lead to radically different EOSs in neutron stars. In particular, new states will reduce the maximum possible neutron star mass which is consistent with a given nuclear EOS.

Simple scenarios where the dark baryons have a mass similar to that of the nucleon and are not charged under a new force do not allow for neutron stars with mass above $\sim 0.7-0.8 M_{\odot}$. This bound is in stark conflict with observation. Charging such dark baryons under a new force with a very light gauge mediator will result in interactions much larger than standard nuclear interactions and can greatly suppress their presence in dense matter. This can mitigate their effect on the EOS enough to allow for neutron stars as heavy as have been observed, $\sim 2 M_{\odot}$. However, if such a new force is similar to nuclear forces as expected in a "mirror world" setup, where the dark neutron has the same selfinteractions as does the visible neutron, the maximum mass is still significantly reduced, and one requires a very stiff highdensity EOS to produce $2 M_{\odot}$ neutron stars. Interestingly, in the case where the dark baryons are stable dark matter, with $m_{\chi} \simeq 938 \mathrm{MeV}$, nuclear strength self-interactions have been implicated to explain dark matter small-scale structure puzzles (see, e.g., [23] and references therein).

Extensions of this work can easily be shown to constrain other possible new weakly interacting particles. For instance, in the "hylogenesis" baryogenesis scenario [2], there are two kinds of baryon-number-carrying dark matter particles, called " $Y$ " and " $\Phi$," which also carry another conserved charge for stability but which have an allowed reaction $n+\gamma \leftrightarrow Y+\Phi$. The stability of matter places a lower bound of $937.90 \mathrm{MeV}$ on $m_{Y}+m_{\Phi}$. The existence of observed neutron stars will place a more stringent bound on $m_{Y}+m_{\Phi}$, which will be similar to the lower bound of $1.2 \mathrm{GeV}$ we found on $m_{\chi}$. Another type of new particle which would be constrained would be a new weak interacting neutral integer spin boson, called " $\xi$," with baryon number 1 and interactions with ordinary matter 
which are not highly suppressed. As long as the lepton number is conserved, both $\xi$ and the proton are stable. The stability of nuclei with atomic number $A$ and charge $Z$ against decays of type $(A, Z) \rightarrow(A-2, Z)+2 \xi$ will place a lower bound of the order of the nucleon mass on $m_{\xi}$. Neutron stars, however, will constrain $\xi$ to be heavier than the minimum chemical potential for neutrons in a twosolar-mass neutron star, or else neutrons could convert to $\xi$ particles and destabilize the star.

As noted earlier, avoidance of such constraints is possible if the dark matter or dark baryon carries sufficiently repulsive self-interactions. If the self-repulsion of the new state is large enough, most of the mass of the star will remain in the form of neutrons, and the effect on the maximum mass will be small.

We thank Brian Batell, Pavel Fileviez Perez, and Maxim Pospelov for helpful discussions. We thank the Institute of Nuclear Theory and the organizers of the INT program Neutron-Antineutron Oscillations: Appearance, Disappearance, and Baryogenesis where this project began. D. M. is supported by PITT PACC through the Samuel P. Langley Fellowship. The work of A. E. N. was supported in part by the U.S. Department of Energy under Grant No. DE-SC0011637 and by the Kenneth K. Young Chair. S. R. and D. Z. are supported by the U.S. Department of Energy under Grant No. DE-FG02-00ER41132.

Note added in proof.-Recently, two groups [24] reached similar conclusions about the maximal mass of neutron stars in the case of neutrons converting into noninteracting dark baryons. Strong self-interactions among the dark baryons as suggested above to evade neutron star limits were further explored in Ref. [25].

*mckeen@triumf.ca

†aenelson@uw.edu

‡sareddy@uw.edu

§zdk@uw.edu

[1] J. Shelton and K. M. Zurek, Phys. Rev. D 82, 123512 (2010); K. M. Zurek, Phys. Rep. 537, 91 (2014).

[2] H. Davoudiasl, D. E. Morrissey, K. Sigurdson, and S. Tulin, Phys. Rev. Lett. 105, 211304 (2010); Phys. Rev. D 84, 096008 (2011).

[3] Z. Berezhiani and L. Bento, Phys. Rev. Lett. 96, 081801 (2006).
[4] D. McKeen and A. E. Nelson, Phys. Rev. D 94, 076002 (2016).

[5] H. Davoudiasl, Phys. Rev. Lett. 114, 051802 (2015).

[6] A. P. Serebrov et al., Phys. Lett. B 663, 181 (2008).

[7] B. Fornal and B. Grinstein, Phys. Rev. Lett. 120, 191801 (2018).

[8] W. Mampe, L. N. Bondarenko, V. I. Morozov, Yu. N. Panin, and A. I. Fomin, Pis'ma Zh. Eksp. Teor. Fiz. 57, 77 (1993) [JETP Lett. 57, 82 (1993)]; A. Serebrov et al., Phys. Lett. B 605, 72 (2005); A. Pichlmaier, V. Varlamov, K. Schreckenbach, and P. Geltenbort, Phys. Lett. B 693, 221 (2010); A. Steyerl, J. M. Pendlebury, C. Kaufman, S. S. Malik, and A. M. Desai, Phys. Rev. C 85, 065503 (2012); S. Arzumanov, L. Bondarenko, S. Chernyavsky, P. Geltenbort, V. Morozov, V. V. Nesvizhevsky, Yu. Panin, and A. Strepetov, Phys. Lett. B 745, 79 (2015).

[9] J. Byrne et al., Phys. Rev. Lett. 65, 289 (1990); A. T. Yue, M. S. Dewey, D. M. Gilliam, G. L. Greene, A. B. Laptev, J. S. Nico, W. M. Snow, and F. E. Wietfeldt, Phys. Rev. Lett. 111, 222501 (2013); J. Byrne and P. G. Dawber, Europhys. Lett. 33, 187 (1996).

[10] A. Czarnecki, W. J. Marciano, and A. Sirlin, Phys. Rev. Lett. 120, 202002 (2018).

[11] J. M. Lattimer and M. Prakash, arXiv:1012.3208.

[12] P. Demorest, T. Pennucci, S. Ransom, M. Roberts, and J. Hessels, Nature (London) 467, 1081 (2010).

[13] J. Antoniadis et al., Science 340, 1233232 (2013).

[14] J. M. Arnold, B. Fornal, and M. B. Wise, Phys. Rev. D 87, 075004 (2013).

[15] Z. Tang et al., Phys. Rev. Lett. 121, 022505 (2018).

[16] J. R. Oppenheimer and G. M. Volkoff, Phys. Rev. 55, 374 (1939).

[17] R. C. Tolman, Phys. Rev. 55, 364 (1939).

[18] A. Akmal, V. R. Pandharipande, and D. G. Ravenhall, Phys. Rev. C 58, 1804 (1998).

[19] S. Gandolfi, J. Carlson, S. Reddy, A. W. Steiner, and R. B. Wiringa, Eur. Phys. J. A 50, 10 (2014).

[20] K. Hebeler, J. M. Lattimer, C. J. Pethick, and A. Schwenk, Astrophys. J. 773, 11 (2013).

[21] S. Weinberg, Nucl. Phys. B363, 3 (1991).

[22] N. Glendenning, Compact Stars: Nuclear Physics, Particle Physics, and General Relativity, Astronomy and Astrophysics Library (Springer, New York, 2000).

[23] S. Tulin and H.-B. Yu, Phys. Rep. 730, 1 (2018).

[24] T. F. Motta, P. A. M. Guichon, and A. W. Thomas, J. Phys. G: Nucl. Part. Phys. 45, 05LT01 (2018); G. Baym, D. H. Beck, P. Geltenbort, and J. Shelton, preceding Letter, Phys. Rev. Lett. 121, 061801 (2018).

[25] J. M. Cline and J. M. Cornell, J. High Energy Phys. 07 (2018), 081. 